\title{
Telescope Array anisotropy summary
}

\author{
M. Fukushima ${ }^{1,2}$, D. Ikeda ${ }^{1}$, D. Ivanov ${ }^{3}$, K. Kawata ${ }^{1}$, E. Kido ${ }^{1}$, J.P. Lundquist ${ }^{3}$, \\ J.N. Matthews ${ }^{3}$, T. Nonaka ${ }^{1}$, T. Okuda ${ }^{4}$, G. Rubtsov ${ }^{5}$, H. Sagawa ${ }^{1}$, N. Sakurai ${ }^{6}$, \\ M. Takeda ${ }^{1}$, R. Takeishi ${ }^{1}$, A. Taketa ${ }^{7}$, G.B. Thomson ${ }^{3}$, P. Tinyakov ${ }^{8}$, I. Tkachev ${ }^{5}$, \\ H. Tokuno ${ }^{9}$, S. Troitsky ${ }^{* 6}$ for the Telescope Array Collaboration. \\ ${ }^{1}$ Institute for Cosmic Ray Research, University of Tokyo, Kashiwa, Chiba, Japan; \\ ${ }^{2}$ Kavli Institute for the Physics and Mathematics of the Universe (WPI); \\ ${ }^{3}$ High Energy Astrophysics Institute and Department of Physics and Astronomy, University of \\ Utah, Salt Lake City, Utah, USA; \\ ${ }^{4}$ Department of Physical Sciences, Ritsumeikan University, Kusatsu, Shiga, Japan; \\ ${ }^{5}$ Institute for Nuclear Research of the Russian Academy of Sciences, Moscow, Russia; \\ ${ }^{6}$ Graduate School of Science, Osaka City University, Osaka, Japan; \\ ${ }^{7}$ Earthquake Research Institute, University of Tokyo, Bunkyo-ku, Tokyo, Japan; \\ ${ }^{8}$ Service de Physique Theorique, Universite Libre de Bruxelles, Brussels (ULB), Belgium; \\ ${ }^{9}$ Graduate School of Science and Engineering, Tokyo Institute of Technology, Meguro, Tokyo, \\ Japan. \\ E-mail: stems2.inr.ac.ru
}

The Telescope Array has accumulated the largest UHECR data set in the Northern hemisphere. We make use of these data to search for large- and small-scale anisotropy of UHECR arrival directions. At small angular scales, we report an update on searches for clustering of events and on correlations with various classes of putative sources. At large angular scales, we revisit, with the new data and with new tests, the localized excess of events known as the TA "hot spot". We review some other studies of the arrival directions and corresponding implications for the nature of UHECR sources.

35th International Cosmic Ray Conference - ICRC2017

10-20 July, 2017

Bexco, Busan, Korea

${ }^{*}$ Speaker. 


\section{Introduction and data used}

Introduction. Understanding the origin of ultra-high energy (UHE; energy $E \gtrsim 10^{19} \mathrm{eV}$ ) cosmic rays (CRs) remains one of the most intriguing unsolved problems of astroparticle physics. Small number of events at highest energies and (generally unknown) deflections of primary particles in cosmic magnetic fields render this task particularly difficult. There are no obvious UHECR sources seen in the sky. Therefore, the search for sources has to rely on statistical methods.

A number of approaches can be followed in this direction. Generally, they can be split into several groups.

Global anisotropy (Sec. 2) studies search for deviations from uniformity at the largest angular scales, that is in the best possible approximation to a full-sky analysis (limited by the field of view for any particular terrestrial experiment). These studies are most effective in case a large population of sources exists, so that individual sources do not show up at small statistics. Examples are provided by numerous faint sources whose spatial distribution follows the matter distribution either in the Galaxy or in the local Universe.

Medium-scale anisotropy (Sec. 3)studies deal with large (of constellation size) spots in the sky. One of possible relevant physical cases is a very few bright nearby sources with large magnetic deflections of cosmic-ray particles.

Small-scale anisotropy (Sec. 4) manifests itself at the angles of order of, or slightly larger than the angular resolution of the experiment. Including searches for small-scale clustering (doublets and triplets) of events and correlations of arrival directions of cosmic-ray particles with positions of potential source candidates, this class of methods are most efficient when the sources are bright enough and not that abundant, while the magnetic deflections are small (e.g. at the highest energies, or in the exotic case of neutral primaries).

Spectral and other anisotropies (Sec. 5) include studies of direction dependence in parameters of the cosmic-ray flux which are beyond the study of just arrival directions of events. They include searches for direction-dependent patterns in the energy spectrum and/or the composition of the UHECR primary particles and give additional information helping to understand the physical reasons for possible deviations of isotropy found in the distribution of arrival directions.

Data. In this paper we examine for anisotropy the Telescope Array (TA) surface detector (SD) data collected in the 9 years of operation (May 2008 - May 2017). TA is a hybrid UHECR detector located in the Northern hemisphere in Utah, USA $\left(39^{\circ} 17^{\prime} 48^{\prime \prime} \mathrm{N}, 112^{\circ} 54^{\prime} 31^{\prime \prime} \mathrm{W}\right)$. The SD array consists of 507 scintillator detectors covering the area of approximately $700 \mathrm{~km}^{2}$ (for details see [1]). The atmosphere over the surface array is viewed by 38 fluorescence telescopes arranged in 3 stations [2]. In this analysis we use the SD event set as the one having by far the largest statistics and a simple (geometrical) exposure.

Most part of this analysis makes use of the special data set prepared for anisotropy studies. Compared to the SD data sets used for the spectrum and composition studies, this "anisotropy set" has relaxed cuts on the zenith angle $\left(55^{\circ}\right.$ versus $\left.45^{\circ}\right)$ and on the distance of the reconstructed shower core position drom the array border (all events with the core inside the array boundary are included, compared to the $1.2 \mathrm{~km}$ distance cut in other sets). We tested that relaxing the cuts in this way does not lead to a significant loss of the data quality. By comparing the thrown and reconstructed arrival 
directions of the simulated data sets, the angular resolution of TA events with $E>10 \mathrm{EeV}$ was found to be approximately $1.5^{\circ}$. Events with zenith angles between $45^{\circ}$ and $55^{\circ}$ have even better angular resolution. The energy resolution of the TA surface detector at $E>10 \mathrm{EeV}$ is close to $20 \%$ [3]. Relaxing the cuts results in a considerable increase of the exposure. The anisotropy set contains 3691 events with energies $E>10 \mathrm{EeV}, 257$ events with $E>40 \mathrm{EeV}$ and 108 events with $E>57 \mathrm{EeV}$. For some of the tests at higher energies we use the looser cuts described in Ref. [4] which give 143 events with $E>57 \mathrm{EeV}$ in 9 years.

In the anisotropy studies the crucial role is played by the exposure function. The exposure of the TA SD detector was calculated by the Monte-Carlo (MC) technique with the full simulation of the detector. It follows from these MC simulations that above $10 \mathrm{EeV}$ the efficiency of the TA SD is $100 \%$.

\section{Global anisotropy}

Coordinate distributions. First, we examine the distributions of the TA events in longuitude and latitude various celestial coordinates, for three energy thresholds fixed in previous studies (10 EeV, $40 \mathrm{EeV}$ and $57 \mathrm{EeV})$. To this end we generate a large $\left(10^{5}\right) \mathrm{MC}$ event set corresponding to the uniform UHECR flux modulated with the TA exposure. We then compare the distributions of the events in the coordinates between the data and in the MC set by the Kolmogorov-Smirnov (KS) test. No significant deviation from isotropy is found in the sets with the energy thresholds of $10 \mathrm{EeV}$ and $40 \mathrm{EeV}$. The highest-energy set with $E>57 \mathrm{EeV}$ shows some deviation from isotropy. The strongest deviation occurs in the supergalactic latitude where some excess of events close to the supergalactic plane is observed (the corresponding histograms are shown in Fig. 1), as would be
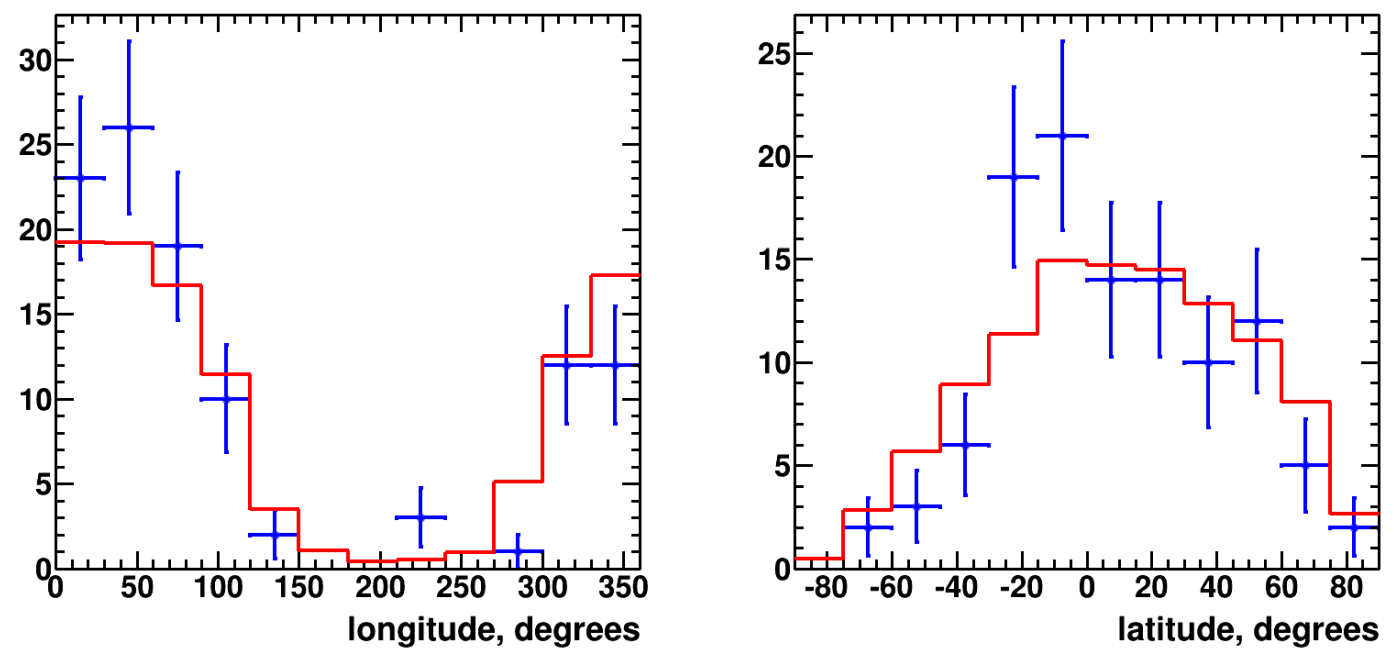

Figure 1: Distributions of events with $E>57 \mathrm{EeV}$ in supergalactic latitude (right panel) and longuitude (left panel).

expected if the UHECRs were produced in the sources following the matter distribution. However, this effect is not statistically significant. 

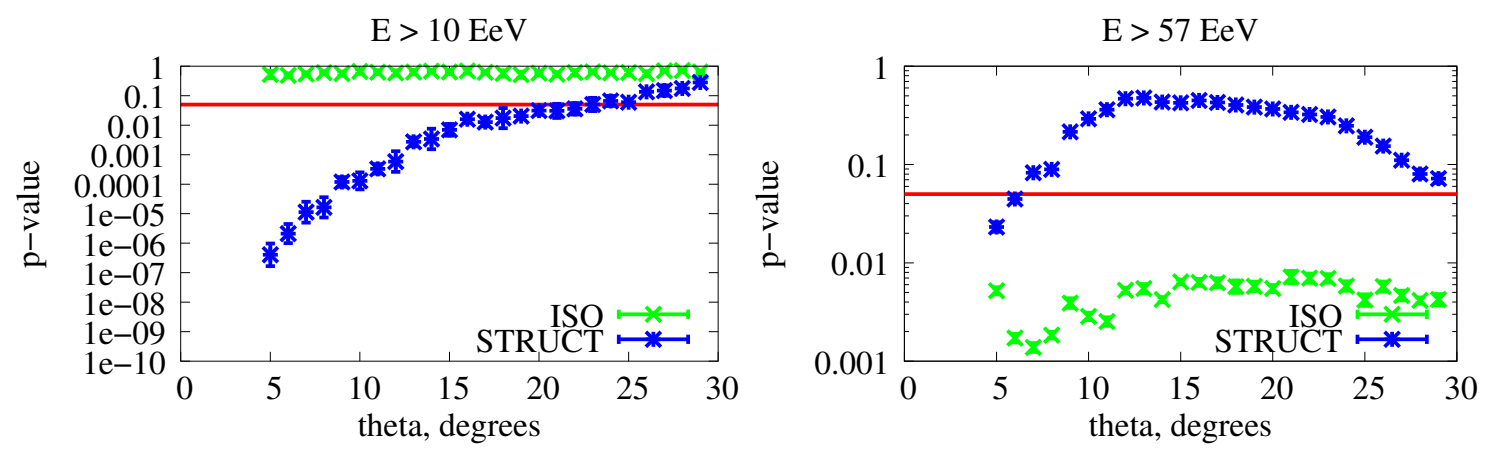

Figure 2: P-values obtained by the flux-sampling test. The blue stars and green crosses correspond to testing the isotropy and the LSS model, respectively. The red horizontal line shows the a priori chosen confidence level of $95 \%$.

Large-scale structure. The UHECR sources, regardless of their nature, are expected to trace the matter distribution. In the limit when the density of sources is sufficiently high so that they can be treated statistically, the UHECR flux can be calculated, as a function of energy, with essentially one free parameter, the typical deflection angle $\theta$. The predicted flux may be compared to observations and thus give constraints on the possible values of $\theta$. The analysis of this type has been previously performed using the HiRes [5], the PAO [6, 7] and the TA [8] data.

We have examined the most recent TA data set for correlations with the LSS. The mass distribution in the Universe was inferred from the 2MASS Galaxy Redshift Catalog (XSCz) that is derived from the 2MASS Extended Source Catalog (XSC). We have assumed that sources follow the matter distribution, and propagated UHECRs from sources to the Earth taking full account of the energy attenuation processes under the assumption that the primary particles are protons. The arrival directions were smeared with the $2 \mathrm{~d}$ Gaussian function of the angular width $\theta$.

The map of the predicted flux was compared to the sky distribution of the observed UHECR events by the parameter-free flux-sampling test (see Refs. [8, 9] for details). At a given value of $\theta$, the result of the test is the $p$-value that shows how likely it is that the UHECR distribution follows the one expected in a given model (LSS or isotropy). The results of the test, as a function of $\theta$, are shown in Fig. 2 for two energy thresholds of $10 \mathrm{EeV}$ and $57 \mathrm{EeV}$ as indicated on the plots. The blue crosses and green pluses show the $p$-values obtained by testing the isotropy and the LSS model, respectively. The red horizontal line shows the confidence level of $95 \%$.

At low energies $E>10 \mathrm{EeV}$, the data are compatible with isotropy and not compatible with the structure model unless the smearing angle is larger than $\sim 20^{\circ}$. This is expected, since even in the case of protons, and taking into account the regular component of the Galactic magnetic field only, the deflections of the UHECR at $E \sim 10 \mathrm{EeV}$ are expected to be of the order of $20-40^{\circ}$, depending on the direction.

At intermediate energies $E>40 \mathrm{EeV}$ (not shown on Fig. 2), the situation is similar. The TA data are compatible with the isotropic distribution and not compatible with the LSS model unless the deflections exceed $\sim 10^{\circ}$.

Finally, at the highest energies $E>57 \mathrm{EeV}$, the behavior is different. The data are compatible with the structure model but incompatible with the isotropic distribution at the $\sim 3 \sigma$ C.L. (pretrial), for most values of the smearing angle. 


\section{Medium-scale anisotropy}

The TA hot spot. In the highest energy set with $E>57 \mathrm{EeV}$ collected during the first 5 years of the TA operation, a concentration of events has been observed in the circle of radius $20^{\circ}$ around the direction $\mathrm{RA}=147^{\circ}, \mathrm{DEC}=43^{\circ}$ [4]. The number of observed events in this "hot spot" was found to be 19 out of 72 total (for the description of the data set used in the hot spot analysis see Ref. [4]), while 4.5 were expected in the case of a uniform background. The post-trial significance of this excess was evaluated to be $3.7 \times 10^{-4}(3.4 \sigma)$. The number of events has been almost doubled now: TA SD years 6-9 brought 71 additional events satisfying the hot-spot analysis criterium. We take advantage of this enlarged statistics to search for a hot spot in the full data set. Since the number of events in the original hot spot, 19, is small, one expects large statistical fluctuations in both the position and the cosmic-ray flux of the derived source; therefore, by making use of a larger data set, we obtain more precise physical information about this excess of events. However, a drawback of this kind of analysis is that we know what we are looking for, the fact which results in hard-to-quantify look-elsewhere corrections to the statistical significance of any observation.

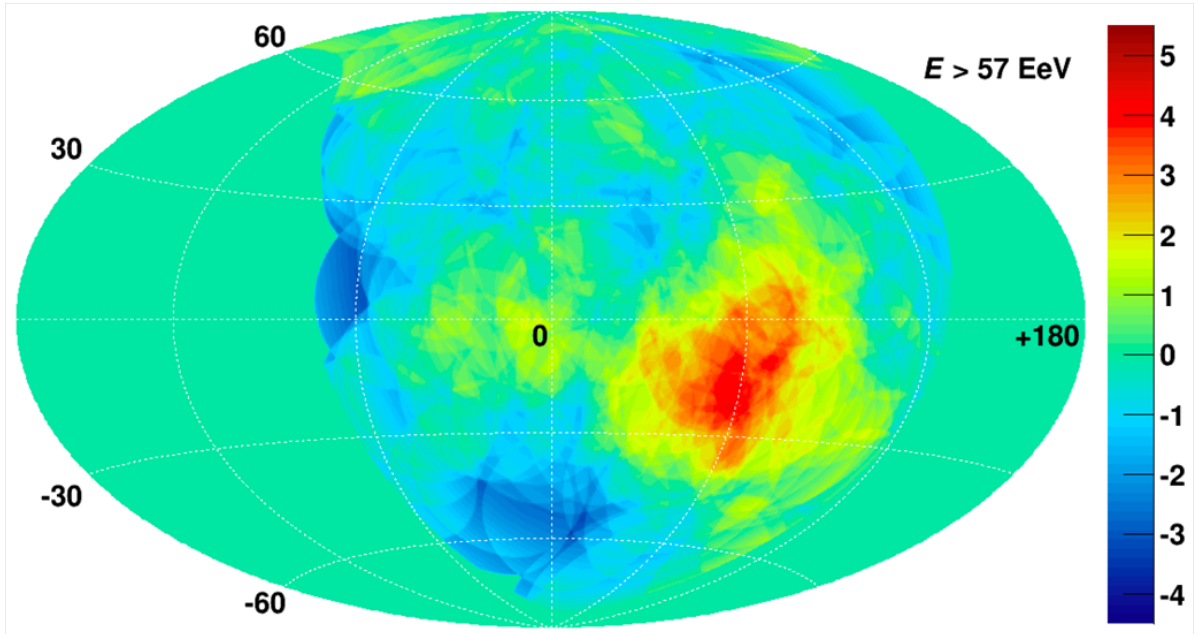

Figure 3: The skymap representing direction-dependent excesses and deficit with respect to the isotropic background, based on 9 years of TA data $(E>57 \mathrm{EeV})$ in supergalactic coordinates.

\section{Small-scale anisotropy}

Clustering. For small angular deflections, individual sources may manifest themselves with doublets, triplets, etc. of events, provided the number of sources contributing to the flux is sufficiently small. In this case, one expects the excess of pairs of events with separations $\delta \sim \sqrt{2} \sqrt{\sigma_{r}^{2}+\sigma_{d}^{2}}$, where $\sigma_{r}$ is the angular resolution of the experiment (more precisely, the opening angle containing $68 \%$ of reconstructed arrival directions, with respect to the true arrival direction) and $\sigma_{d}$ is the magnetic deflection angle. Since $\sigma_{d}$ is in general unknown, a scan over $\delta$ is often performed, and if some deviation from isotropy is found, the look-elsewhere effect is taken into account in the estimation of the statistical significance. This test has been performed at two energy thresholds, $40 \mathrm{EeV}$ and $57 \mathrm{EeV}$. There is a weak deviation from the isotropy at intermediate angular scales in 
the highest-energy data set. This deviation is consistent in angular scale with the one being caused by the TA hot spot [4].

At the highest energies, however, $\sigma_{d}$ is always small, and the expected doublet separation becomes simply $\delta \sim \sqrt{2} \sigma_{r}$, removing the necessity of the scan over $\delta$. This is the case for energies above $100 \mathrm{EeV}$. For these highest-energy events, $\sigma_{r} \approx 1^{\circ}$ for the TA SD, as determined from MC simulations.

Previously, in the very first published data sets of Telescope Array and the Pierre Auger Observatory, one doublet had been found among only 6 events published [10]. More TA data brought another $E>100 \mathrm{EeV}$ event in its vicinity [4]. The 9-year data was searched for doublets at fixed $\delta=1.4^{\circ}$, and one more doublet was found in the Ursa Major constellation. The probability of having two doublets in the data set as a fluctuation of the isotropic distribution is $0.3 \%$.

\section{Spectral anisotropies}

Additional information may be added to the arrival direction study in order to better understand the origin of observed deviations from isotropy. It includes searches of the energy dependence of anisotropy features, which may be, in particular but not exclusively, caused by the energy dependence in magnetic deflections of particles. These searches have been performed with the TA data.

At the global scale, we have searched for potential spectrum dependence from the declination. Though physically the declination is not a singled out coordinate since it is associated with the Earth and not with the distribution of sources, account of potential differences between Northern and Southern sky may help to improve joint analyses of TA and PAO data. This kind of anisotropy has been found and reported at the conference [11].

At the medium scale, we performed two kinds of analysis. The first one compares the event distribution in energy bins between equal-exposure spherical caps in different directions. As discussed in detail in the contribution [12], the scan over the arrival directions demonstrated the maximal spectral anisotropy in the TA hot spot direction, so that the hot spot at $E>57 \mathrm{EeV}$ is accompanied by a "cold spot" at lower $E>10^{19.2} \mathrm{eV}$.

The second analysis, reported in $[13,14]$ determines the "on-source" area on the sky (the hot spot position and the supergalactic plane have been considered), fully reconstructs the spectrum with events in this area and compares it to the spectrum in the "off-source" area (the rest of the sky). The difference in highest-energy spectra has been demonstrated by this method when the Supergalactic plane band was considered as the "on-source" area.

We refer the reader to the cited contributions for more details about the spectral anisotropies.

\section{Summary and conclusions}

In summary, we have examined the 9-year TA SD data set for various possible deviations from isotropy: distributions in the Galactic and supergalactic coordinates, correlation with the LSS of the Universe, medium-scale and small-scale clustering, correlations with AGNs etc. The two sets with $E>10 \mathrm{EeV}$ and $E>40 \mathrm{EeV}$ show no significant deviation from isotropy in any of the performed 
tests. At the same time, the highest-energy set with $E>57 \mathrm{EeV}$ behaves differently and deviates from isotropy in a number of tests.

Acknowledgment: The Telescope Array experiment is supported by the Japan Society for the Promotion of Science through Grants-in-Aid for Scientific Research on Specially Promoted Research (21000002) "Extreme Phenomena in the Universe Explored by Highest Energy Cosmic Rays" and for Scientific Research (19104006), and the Inter-University Research Program of the Institute for Cosmic Ray Research; by the U.S. National Science Foundation awards PHY-0307098, PHY-0601915, PHY-0649681, PHY-0703893, PHY-0758342, PHY-0848320, PHY-1069280, PHY-1069286, PHY-1404495 and PHY-1404502; by the National Research Foundation of Korea (2015R1A2A1A01006870, 2015R1A2A1A15055344, 2016R1A5A1013277, 2007-0093860, 2016R1A2B4014967); by the Russian Academy of Sciences, RFBR grant 16-02-00962a (INR), IISN project No. 4.4502.13, and Belgian Science Policy under IUAP VII/37 (ULB). The foundations of Dr. Ezekiel R. and Edna Wattis Dumke, Willard L. Eccles, and George S. and Dolores Doré Eccles all helped with generous donations. The State of Utah supported the project through its Economic Development Board, and the University of Utah through the Office of the Vice President for Research. The experimental site became available through the cooperation of the Utah School and Institutional Trust Lands Administration (SITLA), U.S. Bureau of Land Management (BLM), and the U.S. Air Force. We appreciate the assistance of the State of Utah and Fillmore offices of the BLM in crafting the Plan of Development for the site. We also wish to thank the people and the officials of Millard County, Utah for their steadfast and warm support. We gratefully acknowledge the contributions from the technical staffs of our home institutions. An allocation of computer time from the Center for High Performance Computing at the University of Utah is gratefully acknowledged.

\section{References}

[1] T. Abu-Zayyad et al. [Telescope Array Collaboration], The surface detector array of the Telescope Array experiment, Nucl. Instrum. Meth. A 689 (2013) 87 [arXiv:1201.4964 [astro-ph.IM]].

[2] H. Tokuno et al., New air fluorescence detectors employed in the Telescope Array experiment, Nucl. Instrum. Meth. A 676 (2012) 54 [arXiv:1201.0002 [astro-ph.IM]].

[3] T. Abu-Zayyad et al. [Telescope Array Collaboration], The Cosmic Ray Energy Spectrum Observed with the Surface Detector of the Telescope Array Experiment, Astrophys. J. 768 (2013) L1 [arXiv:1205.5067 [astro-ph.HE]].

[4] R. U. Abbasi et al. [Telescope Array Collaboration], Indications of Intermediate-Scale Anisotropy of Cosmic Rays with Energy Greater Than 57 EeV in the Northern Sky Measured with the Surface Detector of the Telescope Array Experiment, Astrophys. J. 790 (2014) L21 [arXiv:1404.5890 [astro-ph.HE]].

[5] R. U. Abbasi et al., Analysis of Large-Scale Anisotropy of Ultra-High Energy Cosmic Rays in HiRes Stereo Data, Astrophys. J. 713 (2010) no.1, L64 [arXiv:1002.1444 [astro-ph.HE]].

[6] T. Kashti and E. Waxman, Searching for a Correlation Between Cosmic-Ray Sources Above $10^{19} \mathrm{eV}$ and Large-Scale Structure, JCAP 0805 (2008) 006 [arXiv:0801.4516 [astro-ph]].

[7] F. Oikonomou, A. Connolly, F. B. Abdalla, O. Lahav, S. A. Thomas, D. Waters and E. Waxman, A Search for Correlation of Ultra-High Energy Cosmic Rays with IRAS-PSCz and 2MASS-6dF Galaxies, JCAP 1305 (2013) 015 [arXiv:1207.4043 [astro-ph.HE]].

[8] T. Abu-Zayyad et al. [Telescope Array Collaboration], Search for Anisotropy of Ultra-High Energy Cosmic Rays with the Telescope Array Experiment, Astrophys. J. 757 (2012) 26 [arXiv:1205.5984 [astro-ph.HE]]. 
[9] H. B. J. Koers and P. Tinyakov, Testing large-scale (an)isotropy of ultra-high energy cosmic rays, JCAP 0904 (2009) 003 [arXiv:0812.0860 [astro-ph]].

[10] S. V. Troitsky, A doublet of cosmic-ray events with primary energies $>10^{20} \mathrm{eV}$, JETP Lett. 96 (2012) 13 [arXiv:1205.6435 [astro-ph.HE]].

[11] D. Ivanov [Telescope Array Collaboration], Declination dependence of the Telescope Array Surface-Detector spectrum, PoS(ICRC2017) 496.

[12] J.P. Lundquist [Telescope Array Collaboration], Telescope Array Energy Spectrum Anisotropy, PoS(ICRC2017) 513.

[13] T. Nonaka [Telescope Array Collaboration], Telescope Array spectrum anisotropy, small scale anisotropy and energy correlation, PoS(ICRC2017) 507.

[14] R. U. Abbasi et al. [Telescope Array Collaboration], Search for Anisotropy in the Ultra High Energy Cosmic Ray Spectrum using the Telescope Array Surface Detector, [arXiv:1707.04967 [astro-ph.HE]]. 